\title{
Role of Carboxyamidase in the in vivo metabolism of Chlorfluazuron in the Black Cutworm, Agrotis ipsilon (Hfn.) (Lepidoptera : Noctuidae)
}

\author{
Adel Ramzy Fahmy \\ Entomology Department, Faculty of Science, Ain Shams University \\ Cairo 11566, Egypt \\ Email: arfahmy@link.net
}

\begin{abstract}
Triphenyl phosphate (TPP), a carboxylesterase inhibitor, has synergized chlorfluazuron in the resistant strain of the black cutworm but not in the susceptible strain. In vivo metabolism of ${ }^{14} \mathrm{C}$ - chlorfluazuron revealed that the amount of radiolabeled major metabolites $(2,6$ - difluorobenzoic acid and 2,6 difluorobenzamide) recovered from resistant larvae was four times higher than susceptible larvae. Addition of TPP did not affect chlorfluazuron metabolism pattern in the susceptible insects but it caused a significant reduction of the amount of detected metabolites in the resistant insects. Cleavage of chlorfluazuron molecule took place at one or both of the urea C-NH-C bonds which suggests the responsibility of carboxyamidase enzyme for chlorfluazuron metabolism. In vitro assays revealed that carboxyamidase activity was about four times higher in the resistant larvae than the susceptible ones which supports the hypothesis of the involvement of carboxyamidase in chlorfluazuron resistance in this insect pest.
\end{abstract}

Key words: black cutworm - chlorfluazuron - in vivo degradation - carboxyamidase

\section{INTRODUCTION}

The black cutworm, Agrotis ipsilon (Lepidoptera: Noctuidae) is a serious cosmopolitan pest of corn and several agricultural crops. This noctuid is almost polyphagous that attacks a large number of vegetable crops (Hill, 1983). The control of this pest has become a serious challenge facing applied entomologists nowadays regarding the widening circle of resistance and cross resistance to most available conventional insecticides, including organophosphorus and carbamate insecticides.

Chlorfluazuron, a benzoylphenyl urea compound, is a promising insecticide which is highly effective as a chitin synthesis inhibitor especially on lepidopterous and coleopterous larvae. It is characterized by its unique action, safety to mammals and lack of cross resistance with conventional insecticides (Ishaaya, 1992). Chlorfluazuron has a relatively long half-life in insect bodies with a slow metabolism and elimination rate (Fahmy and Miyata, 1992 and Sammour et al., 2008) which render it as a candidate insecticide for better pest control strategies.

Xenobiotic resistance in insects has evolved predominantly by increasing the metabolic capability of detoxication systems. Carboxylesterases, one of these systems, have been reported as the major metabolizing enzyme of benzoylphenyl urea chitin synthesis inhibitors (Xianchun et al., 2007; Elbert and Nauen, 2000 and Li et al., 1998).

This study aims at clarifying the role of carboxylesterase metabolic enzymes in the in vivo degradation of chlorfluazuron and assessing the in vitro activity of the 
involved esterases in chlorfluazuron susceptible and resistant strains of the black cutworm.

\section{MATERIALS AND METHODS}

\section{Insects:}

Chlorfluazuron resistant and susceptible strains, of the same origin, of the black cutworm, Agrotis ipsilon (Hfn.) were used in this study. The resistance ratio of the resistant strain was more than 100 folds that of the susceptible strain. Rearing technique adopted by Abdin, (1979) was carried out under incubation at a constant temperature $25^{\circ} \mathrm{C}$ and $70 \%$ relative humidity.

\section{Chemicals:}

Chlorfluazuron and ${ }^{14} \mathrm{C}$-labeled chlorfluazuron $(654 \mathrm{MBq} / \mathrm{nmol})$ which was uniformly labeled at the 2,5-difluorophenyl ring were used for larval treatment. Unlabeled proposed chlorfluazuron metabolites i.e. 2,6-difluorobenzoic acid and 2,6-difluorobenzamide along with the parent compound (chlorfluazuron) were separated by thin layer chromatography (TLC) to assess their RF values. Triphenyl phosphate (TPP) (95\% pure) was used as a synergist. Silica gel $60 \mathrm{~F}_{254}$ pre-coated plates were used for TLC and X-ray films were used for tracing radioactivity. Acetyl p-nitroanilide was used as a substrate for assaying the in vitro activity of carboxyamidase.

\section{Synergism study}

Chlorfluazuron was mixed with the same concentration of triphenyl phosphate (TPP) at the ratio of $1: 1(\mathrm{v} / \mathrm{v})$. Castor oil leaves, Ricinus communis were dipped in this mixture or in chlorfluazuron alone, left to air-dry at room temperature then offered to the newly moulted $4^{\text {th }}$ instar larvae.

\section{In vivo metabolism of ${ }^{14} \mathrm{C}$-chlorfluazuron:}

Early $4^{\text {th }}$ instar larvae were previously starved for 24 hours before experiment to ensure a whole consumption of the treated castor oil leaf. Two $\mu 1$ of acetone solution containing $10^{-3 \mathrm{M}}$ of radiolabeled chlorfluazuron, or chlorfluazuron with TPP synergist at $1: 5$ ratio $(\mathrm{w} / \mathrm{w})$, was applied to a small castor oil leaf disc $(3 \mathrm{~mm}$ X $3 \mathrm{~mm})$. They were left to airdry and then put singly to each glass homogenizer. Larvae were then introduced singly to each glass homogenizer. Five larvae with three replicates were used for each treatment. Each leaf disc was totally consumed by each larva within four hours. The five larvae of each test were then collected in one of the glass homogenizers and incubated for 96 hours at $25^{\circ} \mathrm{C}$. The glass homogenizers were then rinsed three times with $1 \mathrm{ml}$ of extraction solvent (acetonitrile : ethyl acetate : methanol : water, $1: 1: 1: 1 \mathrm{v} / \mathrm{v}$ ) and the rinsing solution was added to the glass homogenizer containing the larvae. The larvae were then homogenized in the same solution for one minute at $4^{\circ} \mathrm{C}$ (El-Saidy et al., 1989). The products were extracted three successive times by addition of one $\mathrm{ml}$ of the extraction solvent then centrifuged at $15000 \mathrm{rpm}$ for 5 minutes. The combined extracts were concentrated under vacuum at $35^{\circ} \mathrm{C}$ using a rotary evaporator.

The extract was analyzed by TLC using a developing solvent system (ethyl acetate: toluene: acetic acid, 50:45:5, v/v). The plate was then exposed to X-ray film for one week to trace radioactivity. After that, the silica gel plate was cut to small pieces (5mm width), scratched and introduced to a scintillation glass vial containing $5 \mathrm{ml}$ aqueous counting scintillant, ACS $\mathrm{II}^{\circledR}$ then radioactivity was measured using a liquid scintillation spectrophotometer. Ultraviolet light was used to detect the authentic unlabeled compounds.

\section{In vitro assay of carboxyamidase:}

Fourth instar larvae of black cutworm were homogenized in $0.05 \mathrm{M}, \mathrm{pH} 7.5$ Tris-HCl buffer, centrifuged at $1000 \mathrm{~g}$ and the supernatant of the homogenate was used as enzyme source. The assay of carboxyamidase with acetyl p-nitroanilide as a substrate was performed at $30^{\circ} \mathrm{C}$ (Woods et al., 1979). 


\section{RESULTS}

The synergistic effect of triphenyl phosphate (TPP), a carboxylesterase inhibitor, on chlorfluazuron was tested in chlorfluazuron resistant and susceptible strains of the black cutworm larvae and the results are shown in Table (1). TPP did not exert any remarkable synergistic action in the chlorfluazuron susceptible strain of the black cutworm. On the other hand, addition of TPP in the resistant insects has led to a clear synergistic effect on chlorfluazuron, evidenced by the sharp decline in the $\mathrm{LC}_{50}$ value (from 445 to $48.11 \mathrm{ppm}$ ).

Table 1: Synergism of chlorfluazuron by triphenyl phosphate (TPP) in chlorfluazuron susceptible and resistant black cutworm.

\begin{tabular}{|c|c|c|c|c|}
\hline \multirow[b]{2}{*}{ Insecticide } & \multicolumn{2}{|c|}{ Susceptible strain } & \multicolumn{2}{|c|}{ Resistant strain } \\
\hline & $\begin{array}{l}\mathrm{LC}_{50} \mathrm{ppm} \\
(95 \% \text { c.i. })^{\mathrm{b})}\end{array}$ & $\mathrm{SR}^{\mathrm{a})}$ & $\begin{array}{l}\mathrm{LC}_{50} \mathrm{ppm} \\
(95 \% \text { c.i. })^{\mathrm{b})}\end{array}$ & $\mathrm{SR}^{\mathrm{a})}$ \\
\hline $\begin{array}{l}\text { Chlorfluazuron } \\
\text { (alone) }\end{array}$ & $\begin{array}{c}4.00 \\
(2.90-5.12)\end{array}$ & $\begin{array}{ll}--- \\
\end{array}$ & $\begin{array}{l}445 \\
(290-402)\end{array}$ & ---- \\
\hline $\begin{array}{l}\text { Chlorfluazuron } \\
+ \text { TPP }\end{array}$ & $\begin{array}{c}3.40 \\
(2.61-4.30)\end{array}$ & 1.17 & $\begin{array}{c}48.11 \\
(33.5-51.1)\end{array}$ & 9.24 \\
\hline
\end{tabular}

${ }^{\text {a) }}$ Synergistic ratio $=\mathrm{LC}_{50}$ of chlorfluazuron alone $/ \mathrm{LC}_{50}$ of chlorfluazuron $+\mathrm{TPP}$

b) $95 \%$ confidence interval

In vitro metabolism of $\mathrm{C}^{14}$-chlorfluazuron, with and without the addition of TPP, was also investigated in the larvae of the susceptible and resistant strains of the black cutworm and the results are given in Table (2).

Table 2: In vivo metabolism of $\mathrm{C}^{14}$-chlorfluazuron 96 hours after larval treatment with and without the addition of TPP.

\begin{tabular}{|c|c|c|c|c|c|}
\hline \multirow[b]{3}{*}{ Metabolites } & \multicolumn{5}{|c|}{ Percentage of ${ }^{14} \mathrm{C}$-radioactivity recovered $( \pm \mathrm{SD})$} \\
\hline & \multirow[b]{2}{*}{$\mathrm{RF}$} & \multicolumn{2}{|c|}{ Susceptible strain } & \multicolumn{2}{|c|}{ Resistant strain } \\
\hline & & without TPP & $+\mathrm{TPP}$ & without TPP & + ТPP \\
\hline Polar metabolites & 0.00 & $3.3 \pm 1.1$ & $2.9 \pm 0.8$ & $4.9 \pm 1.4$ & $3.9 \pm 09$ \\
\hline 2,6-difluorobenzoic acid & 0.60 & $2.4 \pm 0.3$ & $1.9 \pm 0.5$ & $8.7 \pm 0.3$ & $1.9 \pm 1.0$ \\
\hline 2,6-difluorobenzamide & 0.68 & $1.6 \pm 0.1$ & $1.2 \pm 0.1$ & $7.9 \pm 0.9$ & $2.0 \pm 0.5$ \\
\hline (Parent) Chlorfluazuron & 0.88 & $91.2 \pm 1.3$ & $92.3 \pm 1.7$ & $74.4 \pm 1.6$ & $90.5 \pm 1.1$ \\
\hline Others & ---- & $1.5 \pm 0.2$ & $1.7 \pm 0.3$ & $4.1 \pm 0.4$ & $1.7 \pm 0.2$ \\
\hline$\%$ Total recovery $\pm \mathrm{SD}$ & & $91.2 \pm 0.7$ & $89.9 \pm 1.1$ & $92.1 \pm 2.0$ & $90.7 \pm 2.2$ \\
\hline
\end{tabular}

The detected amounts of total metabolites recovered from the chlorfluazuron resistant insects were three times higher than those recovered from susceptible ones $(25.6 \%$ and $8.8 \%$, respectively).. The recovered amounts of major metabolites resulting from the cleavage of chlorfluazuron molecule at the urea bond (C-NH-C) by the action of carboxyamidase i.e. 2,6difluorobezoic acid and 2,6-difluorobenzamide were more than four times higher in the resistant insects than in the susceptible ones (16.6\% and $4.0 \%$, respectively). Consequently, the amount of unchanged parent compound (chlorfluazuron) in the susceptible strain was remarkably higher than the recovered amount from the resistant strain $(91.2 \%$ and $74.4 \%$, respectively).

Addition of TPP did not have any remarkable effect on the amounts of major metabolites of chlorfluazuron in the susceptible insects (without TPP $=4.0$ and $+\mathrm{TPP}=3.1$ ). On the other hand, addition of TPP in the chlorfluazuron resistant insects has led to a remarkable decrease in the amount of major metabolites (without TPP $=16.6 \%$ and + TPP $=$ $3.9 \%$, respectively). 
Cleavage of chlorfluazuron molecule took place at the urea bond C-NH-C by the action of carboxyamidase enzyme has resulted in two major metabolites which are shown in Fig. (1).<smiles>O=C(NC(=O)c1c(F)cccc1F)Nc1cc(Cl)c(Oc2ncc(C(F)(F)F)cc2Cl)c(Cl)c1</smiles>

Chlorfluazuron<smiles>O=C(O)c1c(F)cccc1F</smiles>

2,6-difluorobenzoic acid

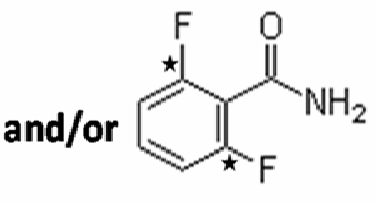

2,6-difluorobenzamide

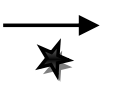

Position of suspected cleavage of chlorfluazuron

Detected major metabolites labeled at the 2,6 position of the difluorophenyl ring

Fig.1 Cleavage of chlorfluazuron molecule at the urea bond by carboxyamidase enzyme.

Carboxyamidase activity from the susceptible and resistant strains of the black cutworm was assayed in vitro using acetyl p-nitroanilide as a substrate. The results or this assay are shown in Table (3).

Table 3: In vitro, carboxyamidase activity in chlorfluazuron susceptible and resistant black cutworm.

\begin{tabular}{lc} 
Strain & $\begin{array}{l}\text { Carboxyamidase activity } \\
\left(10^{-4} \mu \text { mole/min/mg protein }\right)\end{array}$ \\
\hline Susceptible & $1.103 \pm 0.121$ \\
Resistant & $4.129 \pm 1.227$
\end{tabular}

Carboxyamidase activity was about four times higher in the chlorfluazuron resistant strain than the susceptible strain of the black cutworm.

\section{DISCUSSION}

Triphenyl phosphate, TPP is known to be a typical carboxylesterase inhibitor (Ishaaya, 1992 and Haubruge et al., 2002). In the present study, addition of TPP has synergized chlorfluazuron (through inhibition of carboxylesterases) in the resistant insects but not in the susceptible ones. This suggests that carboxylesterases are involved in the mechanism of resistance to chlorfluazuron in the black cutworm. The same pattern of synergist was also reported in chlorfluazuron resistant and susceptible strain of the diamondback moth (Fahmy and Miyata, 1998 and Wu QingJun, 1998). Usmani et al. (2001) have detected $S, S, S$-tri- $n$-butyl phosphorotrithioate (DEF)-sensitive enzymes that hydrolyzed trans-cypermethrin in adults and larvae of the black cutworm. DEF is also known to be an esterase inhibitor.

In order to confirm the role of carboxylesterases as a suspected mechanism of chlorfluazuron resistance in this insect pest, In vivo metabolism of ${ }^{14} \mathrm{C}$-chlorfluazuron was investigated. 2,6-difluorobenzoic acid and 2,6-difluorobenzamide were the major metabolites recovered and detected 96 hours after treating the larvae with ${ }^{14} \mathrm{C}$ chlorfluazuron (Table 2). The amounts of these metabolites were four times higher in the resistant insects than 
susceptible ones. Detected metabolites labeled at the 2,6 positions suggest that cleavage of chlorfluazuron molecule took place at the C-NH-C urea bonds and this strongly supports the hypothesis of involvement of a TPP-sensitive enzyme system (presumably carboxyamidase) in the metabolic breakdown of chlorfluazuron in resistant insects.

Again, addition of TPP did not affect the pattern of chlorfluazuron metabolism in the susceptible strain, but it led to a significant decrease in the amount of major metabolites in the resistant insects due to the fact that TPP inhibits the carboxyamidase enzymes responsible for chlorfluazuron degradation.

The responsibility of carboxyamidase enzyme for chlorfluazuron degradation was further confirmed by the in vitro assay of carboxyamidase activity in chlorfluazuron resistant and susceptible insects using p-nitroanilide as a substrate. Results clearly show that carboxyamidase activity was about four times higher in the resistant insects than the susceptible ones which confirm the same hypothesis.

Only little information is available in the literature on the metabolic breakdown of chlorfluazuron in insect bodies. In most studies on resistance mechanisms, esterases have been confirmed to be involved in resistance by the addition of synergists (esterase inhibitors) or through the in vitro assaying of esterase activity in resistant and susceptible insects (ElSaidy et al., 1998; Haubruge et al., 2002 and Xianchun et al., 2007). In this study the in vivo degradation pattern of ${ }^{14} \mathrm{C}$-chlorfluazuron elucidates the cleavage of chlorfluazuron molecule, metabolites resulted, amount of unchanged parent compound and the involvement of carboxyamidases.

However, it is still necessary to investigate the pattern of metabolite distribution in both larval body and the excreta separately, since the amount of unchanged chlorfluazuron might then show difference due to the speed by which chlorfluazuron reaches its target site inside insect body or excreted with excreta.

\section{REFERENCES}

Abdin, M.I. (1979):. Standard technique for mass rearing of black cutworm, Agrotis ipsilon. M.Sc. Thesis, Fac. of Agric., Al-Azhar Univ., Egypt.

Elbert, A. and Nauen R. (2000): Resistance of Bemisia tabaci (Homoptera: Aleyrodidae) to insecticides in southern Spain with special reference to neonicotinoids. Pest-mgtSci. 56: 60-64.

El-Saidy, M.F., Auda M. and Degheele D. (1989): Detoxification mechanisms of diflubenzuron and teflubenzuron in the larvae of Spodoptera littoralis (Boisd.). Pestic. Biochem. Physiol. 35: 211-222.

Fahmy A. R. and Miyata T. (1998): Joint Action of Chlorfluazuron with Synergists and Insecticides in Chlorfluazuron-Susceptible and Resistant Strains of Diamondback Moth, Plutella xylostella. J. Pesticide Sci. 23: 223-229.

Fahmy A.R. and Miyata T. (1992): Prolonged Effect on Pupae and Adults of the Diamondback Moth, Plutella xylostella (L.) (Lepidoptera: Yponomeutidae) after Larval Treatment with Chlorfluazuron. Appl. Entomol. Zool. 27(3): 349-354.

Haubruge E, Amichot M., Cuany A., Berge J. B. and Ludovic Arnaud (2002): Purification and characterization of a carboxylesterase involved in malathion-specific resistance from Tribolium castaneum (Coleoptera: Tenebrionidae) Insect Biochem. Mol. Biol. 32(9): 1181-1190

Hill, D.S. (1983): Agrotis ipsilon (Hfn.). pp. 357-358. In Agricultural Insect Pests of the Tropics and Their Control, 2nd Edition. Cambridge University Press, Cambridge, London, New York, New Rochelle, Melbourne, Sydney. 746 pages.

Ishaaya, I. (1992): Insect detoxifying enzymes: Their importance in pesticide synergism and resistance. Arch. Insect Biochem. Physiol. 22: 263 - 276

Li TW, Gao XW, Zheng BZ, Zhu SX and Si SY. (1998): A study on carboxylesterase activity in insecticide resistant and susceptible populations of diamondback moth, 
Plutella xylostella, from different regions. Acta Entomologica Sinica 41 (supplement), 26-33.

Sammour E.A., Kandil M.A. and Abdel-Aziz N.F. (2008): The Reproductive Potential and Fate of Chlorfluazuron and Leufenuron Against Cotton Leafworm Spodoptera littoralis (Boisd.). American-Eurasian J. Agric. \& Environ. Sci., 4 (1): 62-67.

Usmani, K. Amin; Knowles, Charles O. (2001): DEF Sensitive Esterases in Homogenates of Larval and Adult Helicoverpa zea, Spodoptera frugiperda, and Agrotis ipsilon (Lepidoptera: Noctuidae). J. Econ. Entomol. 94(4): 884-891.

Woods, M.J., Findlater J.D. and Orsi B.A. (1979): Kinetic mechanism of the aliphatic amidase from Pseudomonas aeruginosa. Biochem. Biophys. Acta 567: 225-237.

Wu QingJun, Zhu GuoRen, Zhao JianZhou, Zhang Xing and Gao XiWu (1998): Resistance selection of Plutella xylostella (L.) by chlorfluazuron and pattern of cross resistance. Acta Entomol. Sinica 41: 34-41.

Xianchun Li, Mary A. Schuler, and May R. Berenbaum (2007): Molecular Mechanisms of Metabolic Resistance to Synthetic and Natural Xenobiotics. Ann. Rev. Entomol. 52: 231-253.

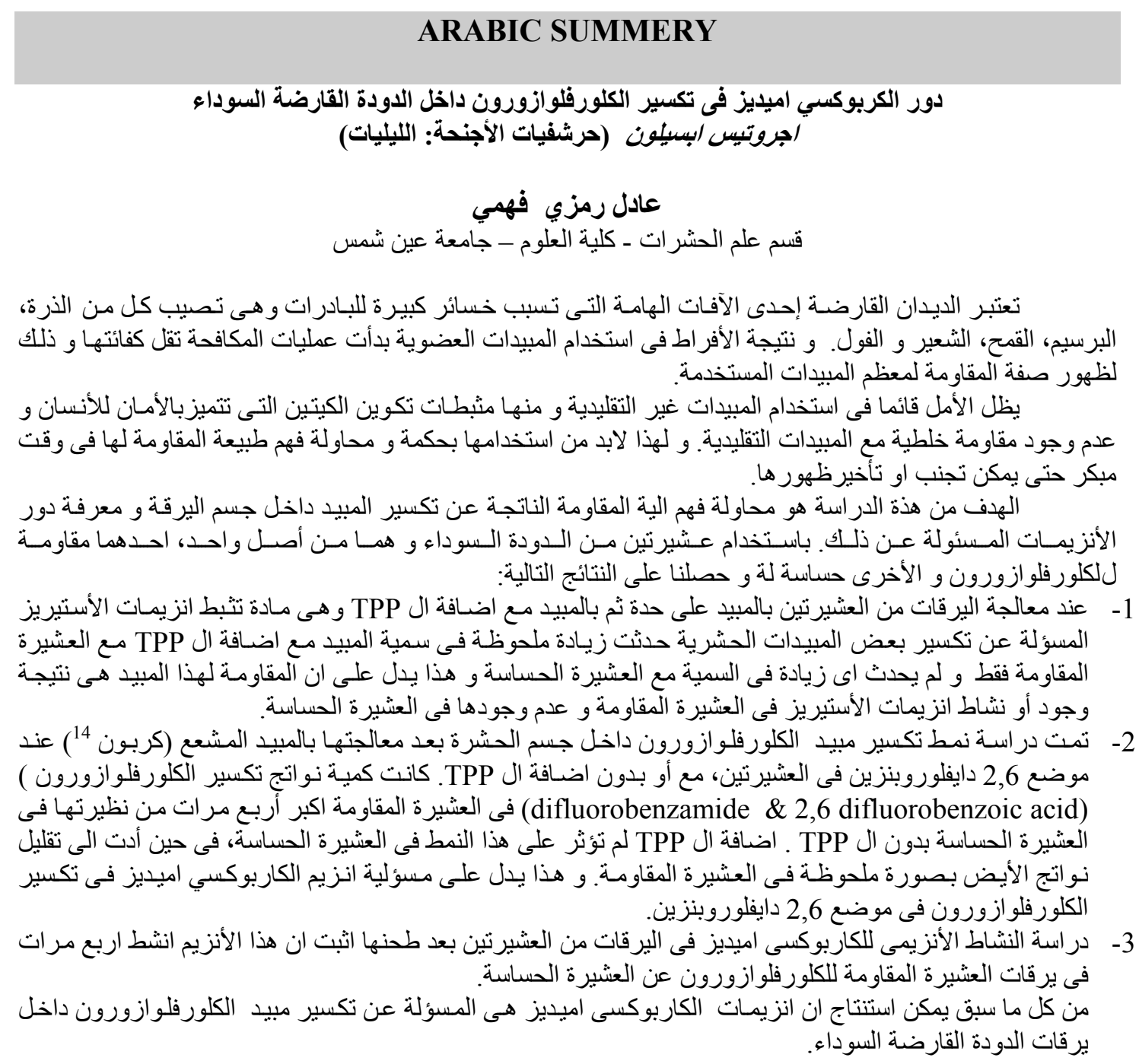

